\title{
THE STRUCTURE OF NUCLEI FAR FROM BETA STABILITY
}

\section{A Progress Report}

by

E. F. Zganjar

Department of Physics and Astronomy

Louisiana State University

Baton Rouge, Louisiana 70803-4001

for the period

May 15, 1991 to May 14, 1992

\section{DISTRIBUTION OF THIS DOCUMENT IS UNLIMITED}

Prepared for the

\section{U. S. Department of Energy}

under

Grant No. DE-FG05-84ER40159

\section{DISCLAIMER}

This report was pripared as an account of work sponsored by an agency of the United States Government. Neither the United States Government nor any agency thereof, nor any of their employees, makes any warranty, express or implied, or assumes any legal liability or responsibility fot the accuracy, completeness, or usefulness of any information, apparatus, product, or process disclosed, or represents that its use would not infringe privately owned rights. Reference herein to any specific commercial product, process, or service by trade name, trademark, manufacturer, or otherwise does not necessarily constitute or imply its endorsement, recommendation, of favoring by the United States Governmer! or any agency thereof. The views and opinions of authors expressed herein do not necessarily state or reflect those of the United States Ġovernment or any agency thereoí. 


\section{HIGHLIGHTS}

(May 15, 1991 - Feb 1, 1992)

- Additional observations of essentially pure $E 0$ transitions in odd-mass nuclei.

- Two on-line nuclear oricntation experiments and four coincidence spectroscopy experiments, led by the LSU group, including two coincidence experiments to search for the $E, I^{*}$ of the superdeformed band in nuclei in the $A=190$ region.

- Six journal publications plus four being prepared for submission.

- Two invited talks at international conferences (EFZ).

- Nine contributions to the proceedings of international conferences.

- The beginning of a strong program in the rare-earth region.

- The development and implementation of a prototype internal pair formation spectrometer.

- The development of a picosecond timing system. 


\section{TABLE OF CONTENTS}

I. INTRODUCTION $\ldots \ldots \ldots \ldots \ldots \ldots \ldots \ldots \ldots \ldots \ldots$

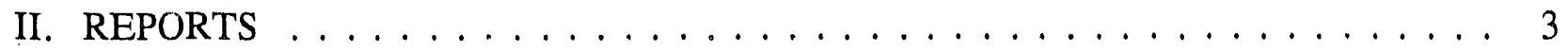

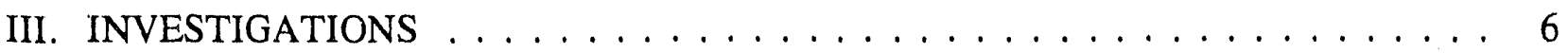

A. The Neutron Deficient $\mathrm{Z}=78-82$ Region $\ldots \ldots \ldots \ldots$

1. Shape Coexistence and Intruder States $\ldots \ldots \ldots \ldots \ldots$

2. The Electric Monopole Transition in Nuclei . . . . . . . . . 7

3. Gold Isotopes . . . . . . . . . . . . . . . . 7

4. Platinum Isotopes $\ldots \ldots \ldots \ldots \ldots \ldots \ldots$

5. Iridium Isotopes $\ldots \ldots \ldots \ldots \ldots \ldots \ldots \ldots \ldots$

6. Search for Superdeformation in ${ }^{19} \mathrm{Hg} \quad \ldots \ldots \ldots \ldots \ldots 14$

7. Search for Population of Superformed States
in ${ }^{194} \mathrm{~Pb}$ Using ${ }^{194} \mathrm{Bi} \beta^{+}$-Decay $\ldots \ldots \ldots \ldots \ldots \ldots$

B. The Neutron Deficient Rare-Earth, $\mathrm{Z}=57-72$ Region $\ldots \ldots \ldots \ldots$

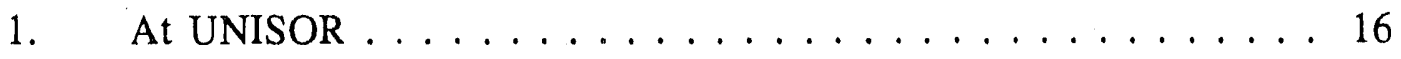

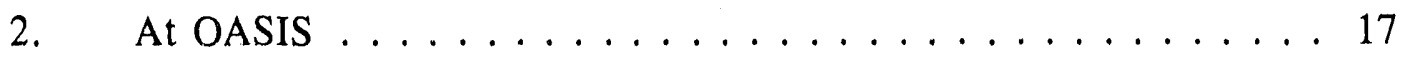

C. The Neutron Deficient $\mathrm{Z}=50-56$ Region $\ldots \ldots \ldots \ldots$

D. Detailed Nuclear Structure Studies Far From Stability . . . . . . . . . 19

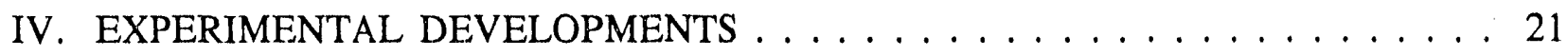

A. Prototype Internal Pair Spectrometer $\ldots \ldots \ldots \ldots \ldots \ldots \ldots$

B. Picosecond Lifetime Spectrometer . . . . . . . . . . . . . 23 


\section{INTRODUCTION}

The research at UNISOR constitutes the major effort of our work on far from stability nuclei. The extensive data from the UNISOR studies on isotopes in the $Z=82$ region has not only established a new region of nuclear deformation and a new class of nuclear structure at closed shells, but may ultimately transform our present understanding of shape coexistence. A most important result of the recent work is the emergence of an extensive region of odd-mass nuclei within which low-energy electric monopole $(E 0)$ transitions occur. This is a new phenomenon in nuclear structure. The implementation of high resolution, high quality, conversion-electron spectroscopy at UNISOR by the LSU group has greatly helped to precipitate this development.

We have clearly established a relationship between low-lying $0^{+}$states in even-even nuclei and the occurrence of shape coexistence in the odd-mass neighbors. A picture is developing of a new type of nuclear structure associated with intruder states and shape coexistence which occurs at low energy near a closed shell when the number of active nucleons of the other type is maximal. This information has come largely from studies at UNISOR of odd-mass $\mathrm{Au}$ isotopes where we see coexisting bands resulting from the couplings to both the normal and to the intruder states.

One of the most exciting developments during the past year has been the on-line, lowtemperature, nuclear orientation experiment on ${ }^{184} \mathrm{Au}$ using the new UNISOR Oxford Instruments ${ }^{3} \mathrm{He}-{ }^{4} \mathrm{He}$ dilution refrigerator. This was the first fully on-line nuclear orientation experiment ever performed in the US. We have been deeply involved in the development of the UNISOR low-temperature nuclear orientation facility. The nuclear orientation (NO) facility is one of the most vital and necessary additions to the arsenal of "weapons" that UNISOR can use to obtain information on nuclear structure. Although UNISOR is somewhat late in entering the on-line NO field relative to several European laboratories, we have the advantage of a well 
developed group of spectroscopists and spectroscopic techniques -- a necessity if one is to take full advantage of the nuclear orientation technique.

The possibility of a new region of stable nuclear deformation around the very neutrondeficient Sm isotopes was pointed out several years ago by Leander. This is a new region of deformation where very little is known about the low-spin states. Extensive in-beam measurements are being done in this region, but such studies locate mainly the high-spin states. As we have shown in the $\mathrm{Z}=82$ region, radioactive decay is unique in its ability to populate the low-spin states, and these are the states most critical to the evaluation of nuclear models. This has been a most difficult region for isotope separator studies because the elements involved are so refractory. Major isotope separator ion-source developments, principally with the UNISOR staff and the Georgia Tech group, have overcome these constraints.

During the past several years the LSU group joined forces with the Maryland group to work on nuclei in the neutron deficient $\mathrm{Z}=50$ - 56 region. 'This interaction developed in a quite natural way since the major emphasis of the experiments proposed by the Maryland group involved high-resolution conversion-electron spectroscopy, the specialty of the LSU group.

We continue to develop, provide for, and maintain the UNISOR conversion-electron spectrometer systems and to construct ion-source parts and other ancillary components for the UNISOR isotope separator. During this reporting period we also constructed and utilized a beam-line station for $\mathrm{e}^{-}-\mathrm{e}^{-}, \mathrm{e}^{-}-\gamma, \mathrm{e}^{-}-\alpha, \alpha-\gamma$ coincidence spectroscopy. This syste.n was used to search for superdeformed states and formed the basis of the prototype internal pair formation spectrometer which was used in the superdeformatinn search in ${ }^{192} \mathrm{Hg}$.

In addition to the UNISOR staff who participated in almost all aspects of these experiments, numerous other UNISOR scientist have in one way or another contributed to the work. During the period reported here, the LSU Experimental Nuclear Structure Group consisted of the principal investigator and three graduate sturlents. 


\section{REPORTS}

Listed below are the reports, papers and abstracts which have emerged during the period of support (May 15, 1991 - January 15, 1992) under grant DE-FG05-84ER40159. Those noted by an asterix were reported in 1990 but appeared in print in 1991 .

DOE/ER/40159-163" "Intruder States, Coexistence, and Approaches to Deformation: The Study of ${ }^{120} \mathrm{Xe}$ and the $\mathrm{N}=66$ Isotones," P.F. Mantica, Jr., B.E. Zimmerman, C.E. Ford, W.B. Walters, D.Rupnik, E.F. Zganjar, H.K. Carter, J. Rikovska and N.J. Stone, In Exotic Nuclear Spectrosopy, ed. W. C. McHar ris (Plenum Press, New York, 1990), p. 495.

DOE/ER/40159-165" "Spectroscopic Techniques in the Study of Radioactive Nuclei Far From Stability: Results for the $\mathrm{Z}=82$ Region," E.F. Zganjar, J.L. Wood, and J. Schwarzenberg, in Exotic Nuclear Spectroscopy, ed. W.C. McHarris (Plenum Press, New York, 1990), p.39.

DOE/ER/40159-166" "Electric Monopole Transitions and Shape Coexistence in Nuclei," E.F. Zganjar and J.L. Wood, Nucl. Phys. A520, 427c (1990).

DOE/ER/40159-167 "The Electric Monopole Transition in Nuclei," E.F. Zganjar, Invited paper, Symposium on Recent Advances in Nuclear Structure Research, American Chemical Society Meeting, Atlanta, GA, April 14-19 (1991).

DOE/ER/40159-169 "Single-particle States in the Rare Earth Region Near the Proton Drip Line," K.S. Toth, D.C. Sousa, J.M. Nitschke K.S. Vierinen, P.A. Wilmarth and M.O. Kortelahti, Phys. Lett. $\underline{B}$-- press).

DOE/ER/40159-170 "Spectroscopy of Proton-Rich Nuclei in the Rare Earth Region," K.S. Toth, J.M. Nitschke, K.S. Vierinen, P.A. Wilmarth, R.B. Firestone, and M.O. Kortelahti, Nuclear Instruments and Methods in Physics Research B 56/57, 484 (1991).

DOE/ER/40159-171 "Shape Coexistence in ${ }^{190} \mathrm{Hg}, "$ M.O. Kortelahti, E.F. Zganjar, J.L. Wood, C.R. Bingham, H.K. Carter, K.S. Toth, J.H. Hamilton, J. Kormicki, L. Chaturvedi and W.B. Newbolt, Physical Review C43, 484 (1991). 
DOE/ER/40159-172 "New Insights from Theory and Experiment into the Multiple Band Structures and Unusually Large Deformations in ${ }^{68} \mathrm{Ge}$ and ${ }^{2} \mathrm{Se}, " \mathrm{~L}$. Chaturvedi, X. Zhao, A.V. Ramayya, J.H. Hamilton, J. Kormicki, C. Girit, H. Xie, Y.-R. Jiang, W.-B. Gao, A. Petrovici, K.W. Schmid, Armand Faissler, N.R. Johnson, C. Backtash, I. Y. Lee, F.K. McGowan, M.A. Riley, J.H. McNeill, M.O. Kortelahti, H.Q. Jin, and R.B. Piercey, presented at the Nuclear Structure in the Nineties conference, Vol. 1 of the proceedings April 23-27, 1990, Oak Ridge, Tennessee p. 80.

DOE/ER/40159-173 "Test of Microscopic Calculation of Multiple Band Structures and Large Deformations in ${ }^{68} \mathrm{Ge}$ and ${ }^{72} \mathrm{Se}, " \mathrm{~L}$. Chaturvedi, X. Zhao, A.V. Ramayya, J.H. Hamilton, J. Kormicki, S. Zhu, C. Girit, H. Xie, W.-B. Gao, J.R. Jiang, A. Petrovicki, K.W. Schmid, A. Faissler, N.R. Johnson, C. Baktash, I.Y. Lee, F.K. McGowan, M.L. Halbert, M.A. Riley, J.H. McNeill, M.O. Kortelahti, J.D. Cole, R.B. Piercy, and H.Q. Jin, Phys. Rev. C43, 2541 (1991).

DOE/ER/40159-175 "Investigation of $A=155$ and $A=151$ Nuclides": Identification of the $155 \mathrm{tM} \mathrm{s}_{1 / 2}$ Isomer and of the ${ }^{155} \mathrm{Yb} \beta$-decay branch, K.S. Toth, K.S. Vierinem, M.O. Kortelahti, D.C. Sousa, J.M. Nitschke, and P.A. Wilmarth, Phys. Rev. C44, 1868 (1991).

DOE/ER/40159-176 "On-Line Nuclear Orientation Study of ${ }^{184} \mathrm{Au}$ ", Y. Xu, K.S. Krane, M.A. Gummin, J.L. Wood, M.M. Jarrio, J.B. Breitenbach, E.F. Zganjar, D. Rupnik, H.K. Carter, P.F. Mantica, and B.E. Zimmerman, S.cond International Conference on On-Line Nuclear Orientation and Related Topics, Oak Ridge, TN, October 16-19 (1991)

DOE/ER/40159-177 "Nuclear Structure Studies of ${ }^{187}$ Ir via On-Line Nuclear Orientation," M.A. Gummin, K.S. Krane, Y. Xu, T. Lam, E.F. Zganjar, J.B. Breitenbach, B.E. Zimmerman, P.F. Mantica and H.K. Carter, Second International Conference on On-Line Nuclear Orientation and Related Topics, Oak Ridge, TN, October 16-19 (1991).

DOE/ER/40159-178 "Detailed Nuclear Structure Studies Far From Stability," J.L. Wood, Johannes Schwarzenberg, E.F. Zganjar, and Dubravka Rupnik, Second International Conference on On-Line Nuclear Orientation and Related Topics, Oak Ridge, TN, October 16-19 (1991).

DOE/ER/40159-179 "The History of UNISOR": 1971-1991, Invited paper, Sympusium on Reflections and Directions in Low Energy Heavy Ion Physics, Oak Ridge, TN, October 14-15 (1991). 
DOE/ER/40159-180 "Pure Electric Monopole Transitions in an Odd-Mass Nucleus," J. Schwarzenberg, J.L. Wood, and E.F. Zganjar, Phys. Rev. $\underline{\text { C (in press). }}$

DOE/ER/40159-181 T. Lam, Master's Thesis, Louisiana State University, 1991. 


\section{INVESTIGATIONS}

A. The Neutron Deficient $\mathrm{Z}=78-82$ Region

1. Shape Coexistence and Intruder States

Shape coexistence and intruder states have become the topic of widespread interest in recent years. Following reviews ${ }^{1,2}$ of the topics some years ago, considerable progress had been made in understanding the relationship between intruder states and shape coexistence and in mapping the systematic features of these structures in nuclei. ${ }^{3,4}$ Two major qualitative characteristics have now clearly emerged: Shape coexisting bands intrude to become lowlying states in nuclei where one kind of nucleon is at or near a close shell (subshell) and the other kind of nucleon is near mid-shell; and the intruding bands mix only weakly with neighboring states.

The task for future work on intruder states and shape coexistence is to establish the detailed quantitative features of the energies of the intruders and to determine the mixing between coexisting states. Some partial answers to the energy systematics are already available. For example, in most cases an overall parabolic energy dependence is observed with a minimum near mid-shell for the "other" nucleon. However, significant portions of all these parabolas are missing, most notably when the iniruder states lie above $\sim 1 \mathrm{MeV}$. This is because the detailed spectroscopy necessary to extract the relevant information increases in difficulty with increasing excitation energy. Also, if the parabola lies far from stability, that is if the mid-shell point lies far from stability, then the experiments require on-line isotope

\footnotetext{
${ }^{1}$ K. Heyde et al., Phys. Repts. 102, 291 (1983).

${ }^{2}$ J. H. Hamilton et.al., Repts. Prog. Phys. 48, 631 (1985).

${ }^{3}$ K. Keyde et al., Nucl. Phys. A466, 189 (1987).

${ }^{4}$ K. Heyde et al., Nucl. Phys. A484, 275 (1988).
} 
separation techniques. Detailed spectroscopy of the sophistication required for such studies is now "advancing" into these far from stability regions.

We report here on the current status of intruder states and shapo coexistence in the $\mathrm{Z} \sim 82, \mathrm{~N} \sim 104$ region. This region is of particular interest because it provides the most extensive example of shape coexistence anywhere on the mass surface. It is also the region where the coexisting states are nearly degenerate over the widest range of nucleon numbers.

\section{The Electric Monopole Transition in Nuclei}

The emission of a $\gamma$-ray in the transition between two nuclear states which both have zero spin and even parity $\left(0^{+}\right)$is forbidden since the photon must carry away one unit of angular momentum. The transition may proceed, however, by the internal conversion process through the interaction of an atomic electron with the charge distribution within the confines of the nuclear volume. Such transitions $\left(0^{+} \rightarrow 0^{+}\right)$have been of limited interest since in those cases only the monopole component is possible and the corresponding $E 0$ matrix element is relatively simple. Of greater interest are the $\mathrm{J}^{*} \rightarrow \mathrm{J}^{*}(\mathrm{~J} \neq 0)$ transitions which exhibit significant $E 0$ strength. Until recently, few transitions of this type were observed (particularly in odd-mass nuclei) and $E 0$ was not expected to compete significantly with $M 1$ and $E 2$. A major change in this picture has occurred as a result of studies of nuclear shape coexistence where a significant enhancement of the $E O$ strength has been observed in the $J^{\pi} \rightarrow J^{\pi}$ transitions between the shape coexisting configurations.

\section{Gold Isotopes}

The analysis of the extensive ${ }^{187} \mathrm{Tl} \rightarrow{ }^{187} \mathrm{Hg} \rightarrow{ }^{187} \mathrm{Au}$, and ${ }^{187} \mathrm{Hg} \rightarrow{ }^{187} \mathrm{Au}$ decays is complete. The data were obtained at UNISOR using the ${ }^{176} \mathrm{Hf}\left({ }^{19} \mathrm{~F}, 8 \mathrm{n}\right){ }^{187} \mathrm{Tl}\left(\beta^{+} \mathrm{EC}\right){ }^{187} \mathrm{Hg}$, and ${ }^{176} \mathrm{Hf}\left({ }^{16} \mathrm{O}, 5 \mathrm{n}\right){ }^{187} \mathrm{Hg}$ reactions. The beta decay of ${ }^{187} \mathrm{~T} 1$ predominantly populates the $3 / 2,{ }^{187} \mathrm{Hg}$ 
ground state $(2.4 \mathrm{~min})$ and, consequently the low-spin states of ${ }^{187} \mathrm{Au}$. The heavy ion reaction preferentially populates the $13 / 2^{+},{ }^{187} \mathrm{Hg}$ isomer $(1.7 \mathrm{~min}$.) and, consequently, more high-spin states in ${ }^{187} \mathrm{Au}$ are populated by the second reaction.

About 440 transitions and 145 levels have been extracted from the data and assigned to ${ }^{187} \mathrm{Au}$. A group of states have been located which correspond to the core-particle couplings: ${ }^{186} \mathrm{Pt}[\pi(2 \mathrm{p}-6 \mathrm{~h})] \otimes \mathrm{h}_{9 / 2}{ }^{+1},{ }^{186} \mathrm{Pt}[\pi(4 \mathrm{~h})] \otimes \mathrm{h}_{9 / 2}{ }^{+1},{ }^{188} \mathrm{Hg}[\pi(2 \mathrm{p}-4 \mathrm{~h})] \otimes \mathrm{h}_{11 / 2}{ }^{-1}$, and ${ }^{188} \mathrm{Hg}[\pi(2 \mathrm{~h})]$ $\otimes \mathrm{h}_{11 / 2}{ }^{-1}$. This was proposed ${ }^{5}$ as a new particle-core coupling with the signature of low-energy $E O$ transitions between the mixed bands.

We have located a group of levels near $2 \mathrm{MeV}$, which correspond to a coupling between a proton and the states in the core with energy greater than the pairing gap; and we have identified a number of transitions, seen in the lower-spin part of the high-spin data, ${ }^{6}$ which lie in the domain of spins of the decay data, such as the transitions: $478 \mathrm{keV}(1167 \rightarrow 689), 417$ $\mathrm{keV}(1233 \rightarrow 689)$, and $455 \mathrm{keV}(1604 \rightarrow 1149)$. We have also located candidates for the $f_{7 / 2}$ band.

The analysis of these data has been the most complete of any odd-A nucleus that we have attempted. We have, on a routine basis, placed transitions with intensities as low as 0.2 relative to 100 for the $233.4-\mathrm{keV}$ transition. The most extensive analysis of multiplets ever attempted has yielded 51 transitions with differences in energy of $1 \mathrm{heV}$ (instrumental resolution, FWHM $=2 \mathrm{keV}$ ), and 42 transitions with energy separations $\approx 0.5 \mathrm{keV}$. A total of 1906 coincidence gates were carefully analyzed to establish coincidence relations, but more importantly, to extract the separate intensities of the multiplets which cannot be separated in the ungated spectra.

5 J.L. Wood, E. F. Zganjar, M.O. Kortelathi, J. D. Cole, and H.K. Carter, Z. Phys. A330, 371 (1988).

${ }^{6}$ C. Bourgeois, M. G. Poquet, N. Perrin, H. Segolle, F. Hannachi, G. Bastin, and F. Beck, Z. Phys. A333, 5 (1989). 


\section{Platinum Isotopes}

\section{Pure Electric Monopole Transitions in ${ }^{185} \mathrm{Pt}$}

Electric monopole $(E 0)$ transitions ${ }^{7}$ occasionally play a role in the decay of excited nuclear states. If the first excited state of a doubly even nucleus has spin parity $J^{x}=0^{+}$, it is the overwhelmingly dominant ${ }^{8}$ decay mode to the $0^{+}$ground state and it takes place by either internal conversion (IC) or internal-pair (IP) formation. Sometimes higher-lying $0^{+}$states decay ${ }^{7}$ by $E 0$ transitions to the ground state in competition with electric quadrupole $(E 2)$ decay to a first excited $2^{+}$state. Very occasionally an excited state with nonzero spin will 'indergo a $\Delta J=0, \Delta \pi=$ no transition to a lower-lying state by a transition with $E 0, M 1$, and/or $E 2$ admixed multipolarities. ${ }^{9}$ In odd-mass nuclei, $E 0$ components in $\Delta J=0, \Delta \pi=$ no transitions are extremely rare. ${ }^{10,11}$ Further, because the spin of any state in a odd-mass nucleus is $\geq 1 / 2, \gamma$ ray emission can always take place. We have evidence for $\Delta J=0, \Delta \pi=n o$ transitions in ${ }^{185} \mathrm{Pt}$ for which we observe only internal conversion, i.e., we do not observe corresponding $\gamma$ rays.

We have studied excited states in ${ }^{185} \mathrm{Pt}$ through the radioactive decay of mass-separated ${ }^{185} \mathrm{Au}\left(6.8 \mathrm{~min} ., J^{*}=5 / 2^{-}\right)$by using the UNISOR isotope separator. The activity was produced by the $\left({ }^{12} \mathrm{C}, 8 n\right)$ reaction on a ${ }^{181} \mathrm{Ta}$ target using $140-\mathrm{MeV}{ }^{12} \mathrm{C}$ ions. Gamma-ray and conversion-electron spectrum multiscaling and $\gamma-\gamma-t, \gamma-x-t, \gamma-\mathrm{Ce}-t$, and Ce- $x-t$ coincidence measurements were conducted on line. Conversion-electron spectra were taken with a $200 \mathrm{~mm}^{2}$

${ }^{7}$ For a review see, e.g., N.A. Voinova-Eliseeva and I. A. Mitropolskii, Fiz. Elem. Chastits. At. Yadra 17, 1173 (1986) [Sov. J. Part. Nucl. 17, 521 (1986)].

${ }^{8}$ Two-photon decay is the only other observed process. For example, in the decay of the first excited state in ${ }^{90} \mathrm{Zr}\left(E=1761 \mathrm{keV}, J^{x}=0^{+}\right)$the relative decay probabilities are $P_{\mathrm{Ic}}: P_{\mathrm{IP}}: P_{2}$ $=1.0: 0.5: 2 \times 10^{-4}$. these data are taken from M. Nessin, T.H. Kruse, and K. E. Eklund, Phys. Rev. 125, 639 (1962) and E.R. Mucciolo and O. Helene, Phys. Rev. C40, 2403 (1989).

${ }^{9}$ See, e.g., J. Lange, K. Kumar, and J. H. Hamilton, Rev. Mod. Phys. 54, 119 (1982).

${ }^{10}$ E. f. Zganjar and J. L. Wood, in Nuclear Structure in the Zirconium Region, Proceedings of the International Workshop, Bad Honef, Germany, 1988, edited by J. Eberth et al. (Springer-Verlag, Berlin, Germany, P.88 (1988)).

${ }^{11}$ C. D. Papanicolopulos et al. Z. Phys. A330, 371 (1988). 
x $3 \mathrm{~mm}$ cooled $\mathrm{Si}(\mathrm{Li})$ detector. All assignments of $\gamma$-ray and internally converted transitions were made on the basis of coincidence information.

Although the absence of observable $\gamma$-decay strength in the reported transitions is surprising, the occurrence of $E 0$ strength in ${ }^{185} \mathrm{Pt}$ is not surprising. Electric monopole strength is expected ${ }^{12}$ whenever configurations with different mean-square charge radii $\left\langle r^{2}\right\rangle$ mix. There is extensive evidence that states with different deformation and therefore different $\left\langle r^{2}\right\rangle$ occur in ${ }^{185-187} \mathrm{Pt}$ : This is supported by energy-level systematics, ${ }^{13} E 2$ transition, probabilities, ${ }^{13,14}$ and isotope shifts $s^{15}\left(\delta\left\langle r^{2}\right\rangle\right)$. We interpret the $E O$ transitions observed in this work as resulting from the mixing of low-lying strongly deformed configurations and higher-lying weakly deformed configurations.

\section{On-Line Nuclear Orientation Study of ${ }^{184} \mathrm{Pt}$}

${ }^{184} \mathrm{Pt}$ has been studied by Finger et al. ${ }^{16}$ and Cailliau et al.,${ }^{17}$ and only the gross features of the coexisting bands have been identified. It is determined from potential energy surface calculations $^{18}$ thiat the ground-state band has a deformation of $\beta=0.22$, corresponding to a

${ }^{12}$ J. Kantele, in Heavy Ions and Nuclear Structure. Proceedings of the Fourteenth Summer School, Mikolajki, Poland, 1984, edited by B. Sikora and Z. Wilhelmi (Harwood Academic, New Your, 1984), p. 391; K. Heyde and R. A. Meyer, Phys. Rev. C37, 2170 (1988).

${ }^{13}$ See, e.g., G. D. Dracoulis et al., J. Phys. G 12, L97 (1986); B. Cederwall et al., Z. Phys. A337, 283 (1990).

${ }^{14}$ U. Garg et al., Phys. Lett. B180, 319 (1986).

${ }^{15}$ H. T. Duong et al., Phys. Lett. B 217, 401 (1989); Th. Hilberath et al., Z. Phys. A332, 107 (1989).

${ }^{16}$ M. Finger, R. Foucher, J. P. Husson, J. Jastrzebski, A. Johnson, G. Asiner, B. R. Erdal, A. Kjelberg, P. Patzelt, A. Hoglund, S. G. Malmskog, and R. Henck, Nucl. Phys. A188, 369 (1972). (1974).

${ }^{17}$ M. Cailliau, R. Foucher, and J. P. Husson, Le Journal De Physique, Tome 35, 469

${ }^{\text {i8 }}$ R. Bengtsson, T. Bengtssor, J. Dudek, G. Leander, W. Nazarewicz, and J.-y. Zhang, Phys. Lett. B183, 1 (1987). 
prolate shape, and the excited secondary minimum occurs at a deformation of $\beta=0.16$ with an oblate shape. The calculation also predicted the band-head energy of the excited band to be around $500 \mathrm{keV}$, which agrees closely with the experimental value $(492 \mathrm{keV})$. In this study, we have re-examined and updated the decay scheme of ${ }^{184} \mathrm{Au}\left(\mathrm{T}_{1 / 2}=53 \mathrm{~s}, \mathrm{I}=6\right) . \quad{ }^{184} \mathrm{Au}$ was produced through the reaction ${ }^{181}{ }^{\mathrm{Ta}}\left({ }^{12} \mathrm{C}, 8 \mathrm{n}\right){ }^{184} \mathrm{Au}$ using a ${ }^{12} \mathrm{C}$ beam of $140 \mathrm{MeV}$. Reaction products were captured and ionized in the ion source of the UNISOR isotope separator operated on-line to the tandem. Both the gamma-ray and conversion electron singles were taken in the multiscaling mode (30 bins/cycle, and $2 \mathrm{~s} / \mathrm{bin}$ ), which permits the determination of half-lives. The $\gamma-\gamma-t, \gamma-x-t$ and $\gamma-e-t$ coincidence data were acquired concurrently with the singles spectra. All assignments of $\gamma$-ray and internal conversion transitions were made on the basis of coincidence measurements.

The nuclear orientation data of ${ }^{184} \mathrm{Au}$ were taken after the spectroscopy experiment. The same reaction was used to produce the ${ }^{184} \mathrm{Au}$ nuclei. Mass-separated activity was extracted from the UNISOR isotope separator, transported through the cold bearn tube, ard implanted at $50 \mathrm{keV}$ into the iron foil soldered onto the cold finger in the UNISOR Nuclear Orientation Facility. The iron foil was polarized by the super-conducting magnet at 0.5 Tesla. To measure the $\gamma$-ray anisotropy, six detectors were placed around the cold finger at positions of $0^{\circ}, 45^{\circ}, 90^{\circ}, 180^{\circ}, 225^{\circ}, 270^{\circ}$ relative to the polarization direction. The data acquisition was organized into 6 cold-warm cycles. For each detector, a total of 400 spectra (each of 5 minutes duration) was collected. The temperature of the cold finger was monitored constantly by observing the anisotropy of the thermometer lines $\left({ }^{57} \mathrm{Co}\right)$. In analyzing the data, spectra in each cold-warm cycle were grouped into cold and warm sum files on the basis of temperature and source position.

Due to the complexity of the decay scheme and insufficient knowledge of the magnetic moment of the parent nucleus $\left({ }^{184} \mathrm{Au}\right)$, all the mixing ratios obtained so far were determined by comparing the anisotropy of the transition of interest with a pure $E 2$ transition originating from 
the same level. Since the degree of orientation is common to both $\gamma$-ray transitions, the unknown $\mathrm{U}_{2} \mathrm{~B}_{2}$ and $\mathrm{U}_{4} \mathrm{~B}_{4}$ can then be canceled out.

The coexistence of two $0^{+}$bands can be clearly seen in the results. The ground-state band is the one with stronger deformation. That is consisteri with the prediction of theoretical calculations. $^{18}$ Another interesting feature of the low-lying level scheme of ${ }^{184} \mathrm{Pt}$ is the appearance of a second set of coexisting bands built on the $2^{+}$states at $649 \mathrm{keV}$ and $1173 \mathrm{keV}$ respectively. From the energy spacing of the bandheads of the second set relative to bands of the first set of coexisting bands, it might be suggested that the second set of bands belongs to the corresponding $\gamma$-vibrational bands of the first set. Enhanced electron conversion for interband $\Delta \mathrm{J}=0$ transitions of both sets of coexisting bands were observed. Heyde $e t$ al. suggested ${ }^{19}$ that the $E O$ transition strength is proportional to the difference of the deformation parameters and mixing amplitude. By studying the energy spacing distortion, Dracoulis et al. predicted $^{20}$ that the mixing of two $0^{+}$bands is strong. Unfortunately, the determination of absolute $E O$ strength requires life-time measurements of the energy levels, which are difficult to obtain. However, the competition of $E 0$ transitions with $M 1$ and $E 2$ transitions and its possible association with band mixing can still be explored.

\section{Iridium Isotopes}

As with the well studied isotopes of $\mathrm{Au}, \mathrm{Pt}$, and $\mathrm{Hg}$, the odd-mass $\mathrm{Ir}$ isotopes around $\mathrm{A}=189$ lie in a transitional region where shapes are changing with increasing mass from prolate to more oblate structures. The level energies very smoothly with decreasing mass until ${ }^{185} \mathrm{Ir}$, where there is a dramatic increase in the two band-head energies, and the $5 / 2$ member of the $h_{9 / 2}$ configuration intrudes to replace that of the [402]3/2 in the ground state. The intrinsic

\footnotetext{
${ }^{19}$ K. Heyde, and R. A. Meyer, Phys. Rev. C42, 790 (1990).

${ }^{20}$ G. D. Dracoulis, A. E. Stuchbery, A. P. Byrne, A. R. Poletti, S. J. Polette, G. Gerl,
} and R. A. Bark, J. Phys. G12, L97 (1986). 
quadrupole moments also increase continuously with decreasing mass until $A=185$, where there is a sizeable prolate deformation. This study of ${ }^{187} \mathrm{Ir}$ is part of a systematic study of the odd iridium isotopes from $\mathrm{A}=185$ to $\mathrm{A}=189$ via nuclear orientation. Special attention has been paid to extracting a consistent set of multipole mixing ratios.

Angular distributions of $\gamma$-rays from the decay of $2.35 \mathrm{hr}{ }^{187} \mathrm{Pt}$ were measured at the online Nuclear Orientation Facility. Reaction products from a ${ }^{176} \mathrm{Hf}$ target and a $125 \mathrm{MeV}{ }^{16} \mathrm{O}$ beam were collected in the UNISOR ion source. The isotopes were then mass separated and implanted at $50 \mathrm{keV}$ into a polished iron foil which was soldered onto the copper cold-finger of the UNISOR ${ }^{3} \mathrm{He}-{ }^{4} \mathrm{He}$ dilution refrigerator. The iron foil was polarized in a $0.6 \mathrm{~T}$ field, providing a hyperfine field of around $128 \mathrm{~T}$ for the orientation of platinum.

Following a preliminary analysis of the orientation data, it became clear that more spectroscopic information was needed in order to complete this analysis. Previous spectroscopic results included many unplaced transitions and observed no transitions at all above 1.3 MeV. The existence of numerous high-intensity multiplets was, howe:'er, most problematic. The occurrence of these complicates analysis of NO data as the angular distributions are then the sum of the transitions involved, and the individual intensities are often poorly known. Poor knowledge of the decay scheme makes a top-down analysis involving deorientation parameters $U_{\lambda}$ very complex, especially when gamma intensity balances are poor. It was also discovered that a great deal of intensity from previously unknown energy lines $(E \geq 2 \mathrm{MeV})$ was populating the low-lying levels.

A short spectroscopy run was performed at UNISOR in which the reaction ${ }^{181} \mathrm{Ta}\left({ }^{12} \mathrm{C}\right.$, $6 n){ }^{187} \mathrm{Au}$ was used to produce ${ }^{187} \mathrm{Pt}$. sixteen hours of $\gamma^{-} \gamma$-t and $\gamma$-e-t coincidences and $\gamma$ and electron multi-scaled singles events were collected with ${ }^{187} \mathrm{Au}$ production rates of over $5 \times 10^{5}$ particles/s. Short counting periods and high rates eliminated the buildup of Ir activity in the spectra, and high-resolution, high-efficiency detectors permitted very good intensity analysis. 
Over $20 \%$ additional more intensity was added to the existing decay scheme, accounting for a total of approximately $82 \%$ of the total $\gamma$-ray intensity.

Mixing ratios for two transitions, 311 and $551 \mathrm{keV}$, were determined by the relative method for $\gamma$-rays in competition with pure $E 2$ transitions. Since at least two transitions from the level at $816 \mathrm{keV}$ have large anisotropies, it can immediately be inferred that the spin of that level is not $1 / 2$, leaving only the $3 / 2$ and $5 / 2$ possibilities. Without a complete top-down analysis, it is not yet possible to find the effective orientation parameter, $\mathrm{B}_{2}$. However, using the pure $E 2$ line at $332 \mathrm{keV}$, it is possible to set a minimum $\mathrm{B}_{2}$ of $0.321 \leq \mathrm{B}_{2} \leq 0.557$, since the product $\mathrm{B}_{2} \mathrm{U}_{2}(443$ level $)=0.439(118)$, and $\mathrm{U}_{2}$ must be less than 1 .

\section{Search for Superdeformation in ${ }^{192} \mathrm{Hg}$}

Superdeformation has been predicted at low spin in the proton-rich $\mathrm{Hg}$ and $\mathrm{Pb}$ regions, ${ }^{21}$ and superdeformed states have been discovered through in-beam reactions in several $\mathrm{Hg}$ isotopes $^{22,23}$ with bands identified down to about $8^{+}$levels. The bandheads and lower spins have not been observed.

We first performed an experiment in August, 1990 to search for superdeformed states in ${ }^{192} \mathrm{Hg}$ with $\mathrm{I} \leq 8$, with the excited states being populater by $\beta^{+}$decay of ${ }^{192} \mathrm{Tl}$. In the first round of analysis no superdeformed states were observed. A more detailed and higher order analysis has been started and is expected to be completed by March, 1992.

In response to our suggestion ${ }^{24}$ that in-beam spectroscopists search for enhanced internal conversion (this could explain why connections between the superdeformed band and the ground

\footnotetext{
${ }^{21}$ P. Bouche et al., Nucl. Phys. A500, 308 (1989).

22 J. A. Becker et al., Phys. Rev. C41, 9 (1990).

${ }^{23}$ D. Ye et al., Phys. Rev. C41, R131 (1990).

${ }^{24}$ E. F. Zganjar and J. L. Wood, Nıcl. Phys. A520, 427 C (1990).
} 
state band are not observed in $\gamma$-ray spectroscopy), Moore et al. ${ }^{25}$ searched for enhanced Xray emission and found none. This prompted us to examine the processes involved in EO transitions, and we discovered that at higher energies (where the superdeformed bands are located), the internal-pair formation process exceeds the internal conversion process; and the internal-pair process does not give rise to $\mathrm{X}$-ray emission.

We conducted an experiment in November 1991 which was designed to be sensitive to the internal pair formation component of $E 0$ transitions (see section IV. A). The results look quite promising and the data are under intense analysis.

\section{Search for Population of Superformed States} in ${ }^{194} \mathrm{~Pb}$ Using ${ }^{194} \mathrm{Bi} \beta^{+}$-Decay

To search for the superdeformed bandhead in ${ }^{194} \mathrm{~Pb}$, we believed the $\beta^{+}$-decay of ${ }^{19} 1 \mathrm{Bi}$ to ${ }^{194} \mathrm{~Pb}$ was optimal for several reasons: First, the $\mathrm{Q}_{\beta}$-value for ${ }^{194} \mathrm{Bi}$ is $7.98 \mathrm{MeV}$, while the spin 0 SD bandhead in the second minimum in ${ }^{194} \mathrm{~Pb}$ is at about $4.8 \mathrm{MeV}$ in excitation; second, ${ }^{194} \mathrm{Bi}$ has two high spin isomers that decay by $\beta^{+}$emission and could populate low spin SD band members that are known clown to spin 6 in ${ }^{194} \mathrm{~Pb}$; and third, Bi can be produced and separated at UNISOR. Thus, based on energy and angular momentum considerations, the study of this decay chain is probably the best to test if $\beta^{+}$-decay can populate a known SD band in the $\mathrm{Hg}$ $\mathrm{Tl}-\mathrm{Pb}$ region, and might provide a method to search for shape isomers in the region.

The ${ }^{124} \mathrm{Bi}$ was produced with a ${ }^{\text {nat }} \operatorname{Re}\left({ }^{16} \mathrm{O}, \mathrm{xn}\right)$ reaction using a beam energy of $170 \mathrm{MeV}$ and a target thickness of approximately $60 \mathrm{mg} / \mathrm{cm}^{2}$. The UNISOR on-line isotope separator was used to separate $A=194$ products from others produced in the reaction. The activity, deposited onto a moving tape, was counted at two counting stations, each with two Ge detectors and a SiLi detector. Gamma-ray and conversion-electron singles and coincidence medsurements were made at each station.

\footnotetext{
${ }^{25}$ E. F. Moore et al., Phys. Lett. B258, 284 (1991).
} 
Careful analyses were made of all the data at each station to search for evidence of the known ${ }^{26} 12 \rightarrow 10,10 \rightarrow 8$, and $8 \rightarrow 6$ SD band transitions in ${ }^{194} \mathrm{~Pb}$ which have energies $0 \mathrm{f} 256$, 213 , and $169 \mathrm{keV}$ respectively. No $\gamma$-rays or conversion electrons from the SD band were observed. The preliminary two sigma upper limits we deduce for population of the spin 12, 10 , and 8 SD band members by $\beta^{+}$-decay are $0.00065,0.00055$, and 0.0015 per decay, respectively. A paper is being prepared for publication of these results.

\section{B. The Neutron Deficient Rare-Earth, $Z=57-72$ Region}

\section{At UNISUR}

Our investigacion of the neutron-deficient rare-earth isotopes with $\mathrm{N}<78$ and $\mathrm{Z}>58$ is continuing at UNISOR. The new thermal ion source developed by the UNISOR/Georgia Tech group was used with the UNISOR mass-separator to produce ${ }^{135} \mathrm{Sm}$ and ${ }^{135} \mathrm{Pm}$ using a 216 $\mathrm{MeV}{ }^{46} \mathrm{Ti}$ beam on a $97.3 \%$ enriched ${ }^{2}$ Mo target. Two detector stations were used to give gamma-gamma and conversion-electron-gamma coincidences and spectrum-multiscaled singles. We collected 14 million gamma-gamma coincidences and about 10 million electron-gamma coincidences. This is a factor of 5 better than the previous UNISOR mass-separated data taken on these isotopes.

\footnotetext{
${ }^{26}$ M. J. Brinkman et al., Z. Phys. A336, 115 (1990) and K. Theine et al., Z. Phys. A336, 113 (1990).
} 


\section{At OASIS}

The decay properties of many short-lived neutron-deficient rare earth nuclei with $65 \leq$ $\mathrm{Z} \leq 71$ were investigated by using the OASIS separator facility, ${ }^{27}$ opcrated on-line at the Lawrence Berkeley Laboratory Super-HILAC. These isotopes were produced in fusion reactions in which targets of ${ }^{96} \mathrm{Ru},{ }^{92} \mathrm{MO},{ }^{94} \mathrm{Mo},{ }^{95} \mathrm{Mo},{ }^{96} \mathrm{Mo}$ and ${ }^{93} \mathrm{Nb}$ were bombarded with ${ }^{64} \mathrm{Zn}$ and ${ }^{58} \mathrm{Ni}$ projectiles. Following mass separation the radioactive products were assayed with a Si particle $\Delta E$-E telescope, a thin plastic scintillator, and a hyperpure and two n-type Ge detectors.

Single-particle states near the 82-neutron shell were delineated, numerous new isotopes, isomers and $\beta$-delayed proton emitters were discovered and the $\alpha$-decay properties of some nuclides with $N \geq 84$ were re-examined. The excitation energies of the $s_{1 / 2}$ and $h_{1 / 2}$ proton states in this mass region were examined, and results on the $\beta$-delayed-proton spectra of ${ }^{145} \mathrm{Dy}$ and ${ }^{147} \mathrm{Er}$ were obtained.

The decay properties of ${ }^{155} \mathrm{Lu},{ }^{155} \mathrm{Yb}$, and ${ }^{155} \mathrm{Tm}$ and of the $\alpha$-decay daughters ${ }^{151} \mathrm{Er}$ and

${ }^{151} \mathrm{Ho}$ were investigated following the one-line mass separation of $\mathrm{A}=155$ nuclides produced in ${ }^{64} \mathrm{Zn}$ irradiations of ${ }^{95} \mathrm{Mo}$. In the study, the half-life of the low-spin isomer in ${ }^{155} \mathrm{Lu}$ was measured to be $140 \pm 20 \mathrm{~ms}$, the $\beta$-decay branch of ${ }^{155} \mathrm{Yb}$ was identified by observing $\beta$ delayed $\gamma$-rays and Tm K X-rays, and the existence in ${ }^{155} \mathrm{Tm}$ of an $\mathrm{s}_{1 / 2}$ isomer $\left(\mathrm{T}_{1 / 2}=44 \pm 4\right.$ $\mathrm{s})$, in addition to the $h_{11 / 2}$ ground state, $\left(T_{1 / 2}=21.6 \pm 0.2 \mathrm{~s}\right)$, was established. Schemes for the $\beta$-decays of ${ }^{155} \mathrm{Yb},{ }^{155} \mathrm{Tm}$, and ${ }^{151} \mathrm{Er}$ were produced. New information on the ${ }^{151} \mathrm{Er}$ decay establishes the $s_{1 / 2}$ isomer in ${ }^{151} \mathrm{Ho}$ to be $41.1 \pm 0.2 \mathrm{keV}$ above the $h_{11 / 2}$ ground state. Also, branching ratios of $90 \pm 5,30 \pm 10$, and $85 \pm 10 \%$ were determined for $\alpha$ decays of ${ }^{155} \mathrm{Yb}$, ${ }^{151} \mathrm{Ho}$, and ${ }^{151} \mathrm{Ho}^{\mathrm{m}}$, respectively.

${ }^{27}$ J. M. Nitschke, Nucl. Instr. and Meth. 206, 341 (1983). 


\section{The Neutron Deficient $Z=50-56$ Region}

One way to determine the configuration of the ground state of a nucleus is to measure the magnetic dipole moment and compare it with the moment calculated empirically from the single-particle moments of neighboring nuclei. Since the moment is sensitive to the angular momenta of the odd particles and hence the configuration of the state in question, it is possible in many cases to distinguish among different configurations, or even estimate the amount of mixing by measuring the moment and comparing it to empirical values.

Analysis has :ecently been completed on the measurement of the magnetic dipole moment of the $3^{+}$ground state of ${ }^{114} \mathrm{Sb}$. Although the ground state of ${ }^{116} \mathrm{Sb}$ is a nearly pure $\pi \mathrm{d}_{5 / 2} \mathrm{VS}_{1 / 2}$ configuration, ${ }^{26,29}{ }^{114} \mathrm{Sb}$ differs in that the position of the $\mathrm{g}_{7 / 2}$ neutron orbital in neighboring ${ }^{113} \mathrm{Sn}$ has been substantially lowered relative to ${ }^{115} \mathrm{Sn}$. Thus, although the $3^{+}$ground state could still arise from the $\pi \mathrm{d}_{s / 2}, \mathrm{vs}_{1 / 2}$ doublet or the $\pi \mathrm{d}_{5 / 2} \mathrm{vg}_{7 / 2}$ multiplet, more likely it is a substantial amount of mixing between states with these different configurations.

The anisotropy of the $\gamma$-rays emitted in the $\beta^{+}$decay of ${ }^{114} \mathrm{Sb}$ were measured on-line as a function of temperature at the UNISOR/NOF Facility and the moment obtained by fitting the anistropy data as a function of inverse temperature. After accounting for incomplete relaxation, a moment value of $1.72(8)$ was obtained from these data, indicating a change in the structure of the ground state of ${ }^{114} \mathrm{Sb}$ from a nearly pure $\pi \mathrm{d}_{5 / 2}, \mathrm{vs}_{1 / 2}$ configuration to one in which there is a large admixture from the $\pi \mathrm{d}_{5 / 2} \mathrm{vg}_{/ / 2}$ configuration. The empirical moment for a $3^{+}$state from this configuration is estimated to be $1.4 \mathrm{n} . \mathrm{m}$., while a value of $2.5 \mathrm{n} . \mathrm{m}$. is expected for a state with the same spin and parity from the $\pi \mathrm{d}_{s_{2 / 2}} \mathrm{Vs}_{1 / 2}$ configuration.

We have recently completed a measurement to determine the ground state spin of ${ }^{114} \mathrm{I}$ by on-line nuclear orientation at the UNISOR/NOF. This was done by measuring the anisotropy as a function of temperature of the $709 \mathrm{keV} 2^{+} \rightarrow 0^{+}$transition in ${ }^{114} \mathrm{Te}$ observed in the decay

${ }^{28}$ Z. Gasci, T. Feynes, Zs. Dombradi, Phys. Rev. C44, 626 (1991).

${ }^{29}$ Z. Gasci, T. Feynes, Zs. Dombradi, S. Brant, V. Paar, Phys. Rev. C44, 642 (1991). 
of ${ }^{114} \mathrm{I}$. Although a temperature dependence was observed, we have found evidence for the existence of a possible isomer in ${ }^{114} \mathrm{I}$ at about $130 \mathrm{keV}$. The lifetime of this level suggests an M3 transition between it and ground. At this time, it is not possible to fit the nuclear orientation data since detailed decay information is required to fit the orientation parameters. Especially important to know is whether the isomer is decaying to feed levels in ${ }^{114} \mathrm{Te}$. We intend to do work which will provide this necessary information.

\section{Detailed Nuclear Structure Studies Far From Stability}

State-of-the-art spectroscopy of nuclei far from stability has achieved an extraordinary level of sophistication and detail in the last ten years. In principle, if a state can be populated, it can be characterized by its energy, spin, parity, and major decay paths. Sometimes its lifetime can be measured. In practice, one is confronted with enormous complexity. To convert raw spectroscopic data into nuclear structure data involves a complex process of disentangling $\gamma$-ray and conversion electron data into decay schemes. Specifically, coincidence techniques, especially coincidence intensities, play a crucial role in this process.

The study of the structure of nuclei remains purely phenomenological: theoretical development is in terms of models with empirically determined parameters. Far from stability these parameters must be extrapolated if predictions are to be made. In consequence there have been surprises such as the large ground-state deformations in the neutron-rich sodium isotopes ${ }^{30}$ and the neutron-deficient mercury isotopes. ${ }^{31}$ These isotopes lie at and near closed shells and "extrapolations" (systematics) and predicted spherical shapes. Thus, the exploration of nuclear structure far from stability relies on careful measurement of as many nuclear properties as possible.

\footnotetext{
¿s C. Thibault, R.Klapisch, C. Rigaud, A. M. Poskanzer, R. Prieels, L. Lessard, and W. Reisdorf, Phys. Rev. C12, 644 (1975). (1972).

${ }^{31}$ J. Bonn, G. Huber, H. J. Kluge, L. Kugler, and E. W. Otten, Phys. Lett. 38B, 308
} 
The study of the excited states of nuclei far from stability provides an excellent guide to the degrees of freedom in nuclei. Two features are highly desirable for such studies. First, the studies need to be as complete as possible, i.e., all levels up to a given excitation energy and spin-parity need to be observed (missing a low-lying level would be a disaster). Second, studies need to be systematic, i.e. all isotopes (or isotones) extending from the stability line to the region far from stability need to be studied. The second criterion is vital for identifying how degrees of freedom change in excitation energy with changing proton or neutron number.

The study of the excited states of nuclei far from stability in a complete (as possible) and systematic way has been a major goal of our experimental program at UNISOR. This is described in detail in DOE/ER/40159-165 and DOE/ER/40159-178, and a glimpse is presented here in Section III.A.3. 


\section{EXPERIMENTAL DEVELOPMENTS}

\section{A. Prototype Internal Pair Spectrometer}

The occurrence of discrete-line superdeformed bands in several regions of the periodic table is now well established experimentally. While most of the superdeformed bands have been found to be in coincidence with transitions between low-spin yrast states in the same nucleus, none of the transitions linking the superdeformed bands with the yrast and near-yrast states have been identified. Several explanations have been put forth to account for this. One proposes that the decay-out process is statistical in nature and that many decay paths share the intensity. ${ }^{32}$ Another suggests that $E 0$ transitions could play an important role. ${ }^{24}$ The latter was tested in-beam recently ${ }^{2 s}$ by looking at the $\mathrm{K}$-shell $\mathrm{X}$-ray yield in coincidence with superdeformed transitions. The rationale underlying this approach is that strong $E O$ transitions would be accompanied by intense ionization in the $\mathrm{K}$-shell. The direct measurement of $E O$ decay in-beam is rather difficult, and thus this indirect technique, although not as definitive as direct spectroscopy, is more suitable to in-beam work. The results ${ }^{25}$ for ${ }^{192} \mathrm{Hg}$ indicate that $E O$ internal conversion is not the mechanism by which the superdeformed bands decay to other state's.

We have shown in the ${ }^{190} \mathrm{Tl}$ decay ${ }^{33}$ that we can, with intense analysis, observe $1 \%$ transitions between coexisting states of moderate prolate and oblate deformation exhibiting strong $E O$ admixtures. If the superdeformed band is populated to this level of intensity, it should be observable if the same decay and detection mechanisms are operative. This is not the case for the superdeformed studizs, however. I the previous investigations where strong EO transitions between shape coexisting bands were observed, ${ }^{33}$ the transition energies were below $\approx 1.5 \mathrm{MeV}$ and were identified by observing the $\mathrm{K}$-shell internal conversion electrons.

\footnotetext{
${ }^{32}$ B. Herskind et al., Phys. Rev. Iett 59, 2416 (1987).

${ }^{33}$ M. O. Kortelahti et al., Phys. Rev. C43, 484 (1991).
} 
At those energies, internal conversion is essentially the only mechanism by which an $E 0$ transition can proceed. At higher energies, however, internal pair formation provides another mechanism for the $E O$ decay -- and one which does not produce a $\mathrm{K}$-shell $\mathrm{X}$-ray. Thus, if internal pair formation were to dominate the process, neither the previous UNISOR measurements $^{34}$ which searched for correlated K-shell electrons, nor the in-beams study ${ }^{25}$ which searched for correlated $\mathrm{K}$-shell $\mathrm{X}$-rays would have observed any $E O$ decay.

By utilizing calculations of theoretical values ${ }^{35}$ of internal-pair to internal conversion probability ratios of $E 0$ transitions for $Z<40$, we are able to predict ratios of internal pair formation to internal conversion probabilities. If the transition energies are above about 3 $\mathrm{MeV}$, one should search for the $E 0$ transitions by correlating the superdeformed band photons with internal pair formation electrons and positrons rather than internal conversion electrons.

Internal-pair spectroscopy will also provide a great deal more sensitivity because of the extra coincidence requirement (3-fold rather than 2 -fold) and because of the angular factor (highest probability when $\mathrm{e}^{+}$and $\mathrm{e}^{\cdot}$ are emitted at 180 degrees). It requires $1.022 \mathrm{MeV}$ of the decay energy to create the $\mathrm{e}^{+} \mathrm{e}^{-}$pair, which then share the remaining energy with the highest probability being that of sharing it equally.

The prototype internal pair spectrometer employed for the ${ }^{192} \mathrm{Hg}$ measurements was assembled from pre-existing components. Two conversion electron spectrometers were placed at $180^{\circ}$ and three HPGe detectors nearby. The system was used in the ${ }^{12} \mathrm{Hg}$ superdeformation search described above.

\footnotetext{
${ }^{34} \mathrm{P}$. Joshi et al., Ph.D. Thesis, Louisiana State University, work in progress.

${ }^{35}$ A. Possoja, Z. Phys., A235, 299 (1986); A. Possoja and T. Solonen, University of Javaskyla, preprint.
} 


\section{B. Picosecond Lifetime Spectrometer}

We reported above on shape coexistence and shell model intruder states in neutrondeticient nuclei in the $\mathrm{Z}=82$ region and on the comparison of measured monopole strength in $Z=50$ nuclei. In the $Z=82$ region, the excitation energy of the $h_{9 / 2}$ intruder shows a parabolic dependence from a high at $\mathrm{N}=126$ to a low at $\mathrm{N}=104$ and a rise again for smaller $\mathrm{N}$. For even-even $\mathrm{Hg}$ isotopes then, the promotion of a pair into this intruder state can give rise to a two-particle, four-hole configuration which comprises the deformed (intruder) configuration. A direct measure of the mixing of co-existing shapes in even-even nuclei is the $E 0$ strength from the partial half-lives of $0^{+}$levels. In order to have the capability to measure these lifetimes at UNISOR, a system is being fabricated to cover the range of $10^{-9}$ to $10^{-11}$ seconds. The system, similar to one designed ${ }^{36}$ at BNL/TRISTAN, will collect the timing information from a triple coincidence between a plastic scintillator, $\mathrm{a} \mathrm{BaF}_{2}$ crystal and a germanium detector which is used to detect $\gamma$-rays. A prototype system was built with borrowed detectors and was successfully tested. When operational, the system will be unique since, unlike the one at BNL, it will be capable of detecting and gating on X-rays and conversion elections as well as $\beta$ particles. This provides more flexibility in measuring lifetimes of levels which are fed or depopulated by decay processes other than $\beta$-emission.

${ }^{36}$ H. Mach, R. L. Gill, and M. Moszynski, Nucl. Inst. Meth. A280, 49 (1989). 

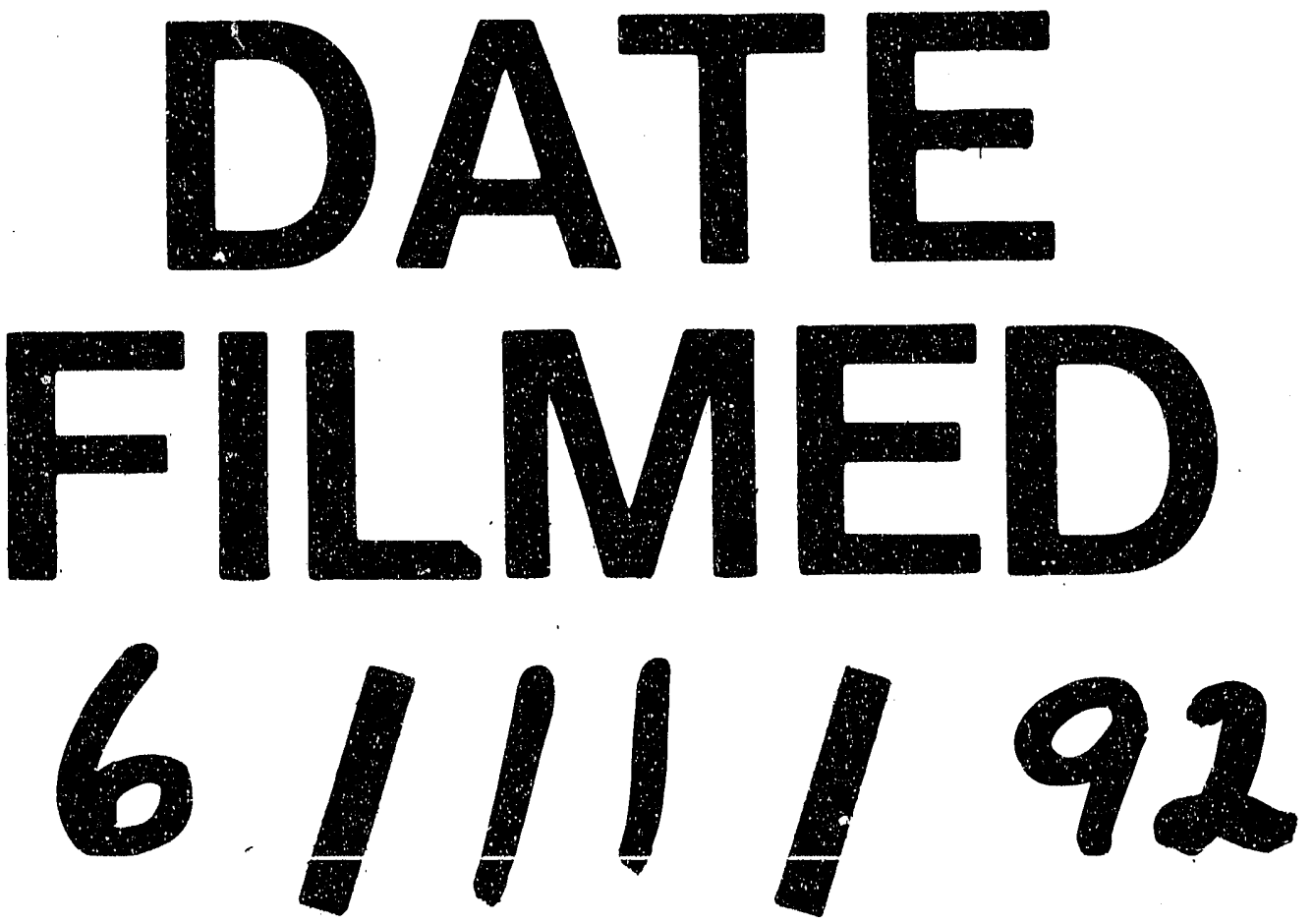
$\frac{}{1}$ 